\title{
Avalanche Delay and Dynamic Triggering in GaAs-Based S-Diodes Doped With Deep Level Impurity
}

\author{
Ilya A. Prudaev ${ }^{\circledR}$, Sergey N. Vainshtein, Maksim G. Verkholetov ${ }^{\circledR}$, \\ Vladimir L. Oleinik ${ }^{\circledR}$, and Viktor V. Kopyev ${ }^{\circledR}$
}

\begin{abstract}
The article is concerned with a detailed switching delay effect exhibited by avalanche S-diodes-superfast GaAs closing switches doped with deep Fe centers. The current and voltage time dependences are simulated in a simplified generator. The dynamic electric field and charge profiles in the structures are calculated. This article describes an impact that Fe capture cross sections of free charge carriers have on delayed switching. The simulation results show that delayed switching is associated with deep center recharging in a double injection mode due to three different processes. There are two different delay mechanisms to be herewith distinguished. A delay effect is experimentally viewed to control the dynamic switching voltage (and the avalanche breakdown voltage) using constant voltage adjustment capability enabled by a triggering circuit supply. The authors demonstrate the way it is possible to adjust the amplitude of current nanosecond pulses in the range of 20-45 A through a lidar transmitter circuit with a semiconductor laser and nonoptimized S-diode. The findings are consistent with the results of numerical simulation.
\end{abstract}

Index Terms - Avalanche breakdown, diodes, microwave switches, optical pulse generators, pulse power systems.

\section{INTRODUCTION}

$\mathbf{R}^{\mathrm{H}}$ ECENTLY, there has been intensive development of LiDARs based on semiconductor lasers designed to be applied in unmanned vehicles (UVs) and advanced driver-assistance systems (ADASs). This is done to address several challenges, one of which implies manufacturing a compact generator of extremely short current pulses for laser diode pumping. Circuits with silicon MOSFET switch

Manuscript received October 17, 2020; accepted November 15, 2020. This work was supported by the Russian Foundation for Basic Research (RFBR), under Project 20-08-00141. The review of this article was arranged by Editor M. A. Pavanello. (Corresponding author: Ilya A. Prudaev.)

Ilya A. Prudaev is with the Department of Semiconductor Electronics, Tomsk State University, 634050 Tomsk, Russia, and also with the Avalanche Electronics LLC, 634034 Tomsk, Russia (e-mail: funcelab @ gmail.com).

Sergey N. Vainshtein was with the University of Oulu, FI-90014 Oulu, Finland. He is now with the Institute of High Pressure Physics PAS, 01-142 Warsaw, Poland.

Maksim G. Verkholetov, Vladimir L. Oleinik, and Viktor V. Kopyev are with the Department of Semiconductor Electronics, Tomsk State University, 634050 Tomsk, Russia.

Color versions of one or more figures in this article are available at https://doi.org/10.1109/TED.2020.3039213.

Digital Object Identifier 10.1109/TED.2020.3039213 provide pulses for a duration of 5-10 ns and an amplitude of 20-30 A [1]. Silicon thyristors are too slow for such tasks, while high-speed, state-of-the-art GaAs thyristors demonstrate 12 A for a duration of $1.4 \mathrm{~ns}$ [2]. The mainstream in the development of these technologies is the use of GaN fieldeffect transistor (FET) and high-electron-mobility transistor (HEMT) transistors. For the simplest resonant circuits with $\mathrm{GaN}$ transistors, durations of 2-2.5 ns were achieved at currents of 20-30 A [3] (more advanced and expensive GaN HEMT modules demonstrate $31 \mathrm{~A}$ at $0.9 \mathrm{~ns}$ [4]).

In well-known resonant circuits for semiconductor laser pumping, a current rise is limited by the reactance: capacitance $(C)$ and inductance $(L)$. When the $L C$-circuit is closed, current oscillations begin with a period of $2 \pi \cdot(L C)^{1 / 2}$. Therefore, it is generally impossible to obtain a pulse with a duration of less than 2.1.(LC $)^{1 / 2}$ (at a half maximum). Thus, a limiting factor is not the speed of voltage commutation of the transistor, but the parasitic inductance of the circuit, which, in turn, is determined by the size of the elements and interconnections. The shortest length of $\mathrm{GaN}$ transistors used for LiDARs exceeds $2 \mathrm{~mm}$ [3]. In fact, these transistors are the largest elements in the $L C$ circuit considered. That is why, in order to reduce parasitic inductance, it is important to use new types of switches. An example of such devices are superfast GaAs switches, which are switched due to the formation of current filaments with a diameter of 20-30 $\mu \mathrm{m}$ and a current density of up to $10 \mathrm{MA} / \mathrm{cm}^{2}$ [5]-[7]. The work will show that such a high current density makes it possible to develop generators of current pulses with an amplitude of up to $45 \mathrm{~A}$ and a duration of $1.1 \mathrm{~ns}$ with an optical power of $135 \mathrm{~W}$.

An avalanche $S$-diode refers to a semiconductor closing switch with an $S$-type current-voltage characteristic. Earlier a switching mechanism of the said device was supposedly attributed to the generation of collapsing field domains (CFDs) in an avalanche regime [8], [9]. The CFD phenomenon has been found from physics-based numerical and experimental studies of superfast switching transient in powerfully avalanching GaAs bipolar transistors [5]-[7] and claims to explain superfast switching in all GaAs devices discussed in the literature over the past 30 years. The CFD effect is actually a bipolar (in the presence of both electrons and holes) Gunn effect that occurs under extreme ionization in the domains exceeding the ionization threshold in amplitude by a factor of $\sim 3$. With time, 


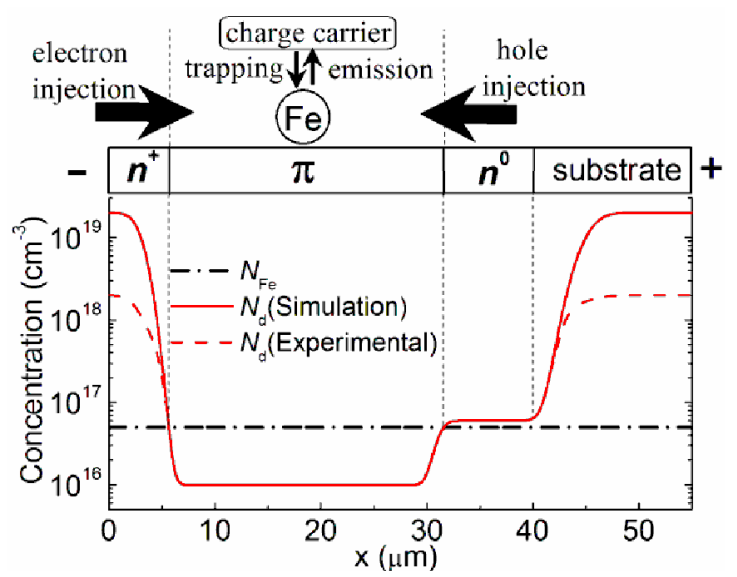

Fig. 1. Doping profiles across the $S$-diode structure. For the simulation, the concentration of $N_{d}$ was increased by one order in $n^{+}$-regions (solid red line) as it was suggested for simulation of CFD in [6]. A sketch of the structure is shown at the top of the figure.

the domains shrink down to $\sim 50 \mathrm{~nm}[5]-[7]$, and then collapse. There is no analytical approach to describe this process, which makes questionable the applicability of Kroemer's criterion for the formation of CFDs threshold. In this work, we concentrate on the peculiarities of the initial avalanche stage of electron-hole plasma formation in $S$-diode, while superfast switching associated with CFDs will be described elsewhere.

The $S$-diode switching voltage is commonly known to rely on the $d V / d t$ voltage ramp applied [9]-[11]. Thus, given that $d V / d t$ increases up to $10^{10}-10^{11} \mathrm{~V} / \mathrm{s}$, the switching voltage can increase by 3-5 times. This effect is attributed to a delayed switching time. This behavior is opposite to a commutation $d V / d t$-effect in thyristors [12], which results in the decreased switching voltage, as the voltage ramp increments, and which is observed in other superfast switches, otherwise referred to as GaAs avalanche bipolar junction transistors (ABJTs) [5], [6]. The switching delay in $S$-diodes is of great practical importance. The article shows that the said effect can increase the dynamic switching voltage (and avalanche breakdown voltage) up to $200-250 \mathrm{~V}$ for the structures with a moderate doping level (donor concentration is about $10^{16} \mathrm{~cm}^{-3}$ ). It will be shown herein that delayed switching exhibited by $S$-diodes provides an efficient circuit with an adjustable output pulse current.

The switching delay mechanism was previously associated with deep-level recharging in the $S$-diode structures [11], [13], [14]. Beloborodov et al. [13], [14] hypothesized that this recharging existed at the avalanche breakdown stage when avalanche-generated holes were trapped by deep acceptors in the $n$-type region of the $S$-diode. This article shows that the above mechanism is not realistic. The simulation results indicate more complicated dynamic recharging of deep centers caused by electron injection, avalanche breakdown, and thermal emission of holes from deep level acceptors. In this case, the injection into the active region of the $S$-diode occurs from opposite sides: the electrons are injected from the forward-biased electron-hole junction, whereas the holes are injected during impact ionization in the reverse-biased junction (the sketch of the $S$-diode is presented in Fig. 1, Section II).

\section{Avalanche S-Diode Structure}

Earlier, $S$-diodes were fabricated based on $n^{+}-\pi-n^{0}-n$ structures where the $n^{0}$-layer (often called $v$-type layer) was codoped with deep level acceptors $(\mathrm{Cr}, \mathrm{Fe}, \mathrm{Mn}$, or $\mathrm{Cu}$ [11]). Presumably, the said $n^{0}$-layer was required to ensure $S$-diode functioning, since it was the very layer to feature the transport carrier process responsible for switching. However, the authors showed in the recent article that the presence of such an $n^{0}$-layer was completely optional with superfast switching to be also found in the structures with a linear $\pi-n$-junction [9]. Despite this, the present article addresses the $n^{+}-\pi-n^{0}-n$ structure in order to show the insignificance of the processes to exist in the $n^{0}$-layer (in this sense, the said experimental $S$-diodes based on $n^{+}-\pi-n^{0}-n$ structures were not optimized). Unlike the previous work [9] to deal with the diffused structures, the current structures were grown through hydride vapor-phase epitaxy (HVPE) so as to obtain more perfect and uniform samples in terms of doping and thickness. This made it possible to achieve better agreement between the experimental and calculated switching voltages of $S$-diodes.

The distribution of dopant concentrations in the structure and its schematic is shown in Fig. 1. The concentration of a shallow donor (sulfur) in the $\pi$-type region was lower than that of a deep $\mathrm{Fe}$ acceptor $\left(N_{\mathrm{Fe}}>N_{d}\right)$. The opposite was true for $n^{0}$-layer $\left(N_{\mathrm{Fe}}<N_{d}\right)$. The thickness of the $\pi$ layer was $d_{\pi}=26 \mu \mathrm{m}$ (the equilibrium hole concentration was $1 \times$ $\left.10^{11} \mathrm{~cm}^{-3}\right)$. The thickness of the $n^{0}$-layer was $d_{\mathrm{n} 0}=8 \mu \mathrm{m}$ (the equilibrium electron concentration was $1 \times 10^{16} \mathrm{~cm}^{-3}$ ). The experimental switching voltage was $V_{s}=58 \pm 13 \mathrm{~V}$, which is close to the theoretical avalanche breakdown voltage of p-n-junction $\left(V_{b}=70 \mathrm{~V}\right.$ for $n=10^{16} \mathrm{~cm}^{-3}$ [15]). Static current-voltage characteristics show that the avalanche breakdown of the compensated $n^{+}-\pi-n^{0}-n$ structure occurs at a voltage of $V=70 \mathrm{~V}$. The calculations were based on Poisson's and continuity equations within the hydrodynamic approximation for charge carriers, using commercial software Technology Computer-Aided Design (TCAD) Synopsys. The detailed calculation models will be presented in Section IV.

Mesa $S$-diode chips were manufactured using standard GaAs processing technology. The ohmic AuGe contacts were made on both sides of the structures. The $n^{+}$-contact area was $1.5 \mathrm{~mm}^{2}$.

\section{S-Diode Operation in Pulse Generators}

The generator circuit is presented in Fig. 2(a). The capacitor $C 1$ is charged with a commercially available $\mathrm{Si}$ MOSFET (VT) with a drain-source voltage of several hundred volts and a rise time of 30-50 ns (this requirement provides a sufficient voltage ramp of about $10^{10} \mathrm{~V} / \mathrm{s}$ ). This MOSFET is closed by a gate pulse that lasts about $100 \mathrm{~ns}$. When the voltage across the capacitor $C 1$ equals the switching voltage of the $S$-diode (SD), the latter closes within a time that is less than $1 \mathrm{~ns}$ with a current pulse being generated in the circuit $L 1-\mathrm{LD}-C 1-\mathrm{SD}$. The parameters of the current pulse depend on resonant circuit parameters [3]. The $C 1$ charging potential can be measured with standard oscilloscope probes with a bandwidth of several hundred $\mathrm{MHz}$ (Tektronix TDS 3052B was used in the work). On the other hand, it seems far more 


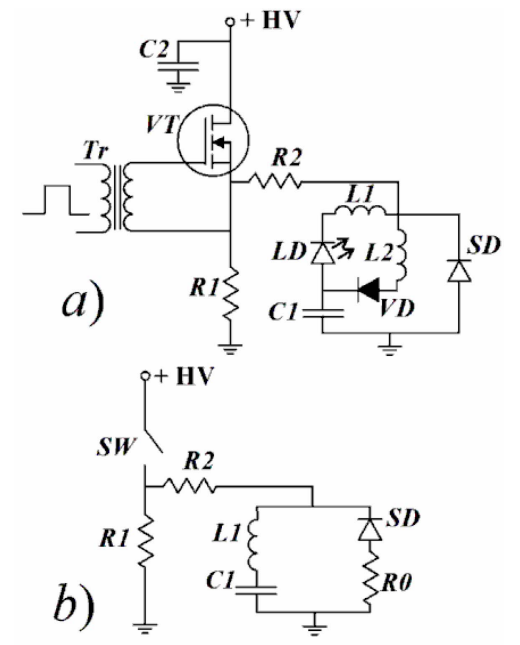

Fig. 2. Electrical circuits used in the experiment (a) and simulation (b): $R 0=1 \Omega, R 1=100 \mathrm{k} \Omega, R 2=30 \Omega, C 1=200 \mathrm{pF}, C 2=100 \mathrm{nF}$, $L 1=1 \mathrm{nH}$, and $L 2=15 \mathrm{nH}, \mathrm{HV}$ - high voltage source connection (up to $250 \mathrm{~V}$ ).

challenging to measure current pulse with a duration of $1 \mathrm{~ns}$ and an amplitude of several tens of amperes. To overcome this obstacle, the optical power of LD was measured (the efficiency of 3-3.6 W/A for this commercial laser chip is known from datasheet [16]). This was achieved through a New Focus $1014(45 \mathrm{GHz})$ high-speed photodetector and a Newport power meter. The shape of the pulses was recorded using a Tektronix DPO 70804C oscilloscope $(8 \mathrm{GHz})$.

The experimental dependences of $S$-diode voltage and optical power-on time for three different $d V / d t$ are shown in Fig. 3. As can be seen, as soon as the transistor opens (OFF mode), the voltage across the $S$-diode slowly decreases with time (e.g., for curve HV2, $110<t<400 \mathrm{~ns}$ ). This decline is attributed to the discharge of the capacitor $C 1$ through the resistor $R 1$ and the $S$-diode (Section V will depict that $S$-diode leakage can be as high as $0.1-0.5 \mathrm{~A}$ at the stage of voltage ramping). As soon as the $S$-diode closes (ON mode), the voltage drops sharply to $0 \mathrm{~V}$ with a short optical pulse to be generated due to the resonant discharge of the capacitor $C 1$ through the laser diode, LD. Thus, the current amplitude can be estimated using a datasheet for LD efficiency (right axis, Fig. 3(b). Obviously, increasing the drain-source voltage, or, essentially, voltage ramp, leads to a reduced switching delay and, concurrently, to the improved switching voltage and pulse current. This is precisely the said effect of delayed switching in avalanche $S$-diodes. Note, the well-known avalanche sharpening diodes operating at higher ramps (above $10^{12} \mathrm{~V} / \mathrm{s}$ ) also establish a characteristic switching delay effect [17]. However, the nature of their delay is associated with the absence of free charge carriers that initiate an impact ionization wave in these superfast switches [18].

To simulate the transient voltage and current relationships in GaAs $S$-diodes, it is reasonable to use a deep center model along with hot-carrier mobility and filament-switching models [5], [6]. Hereby, the simulation was supported by TCAD software that is widely used for the design and simulation of a variety of semiconductor devices. Earlier, commercially available TCAD was successfully used for CFD simulation in superfast GaAs ABJT [5], [6] and GaAs high-gain (HG)
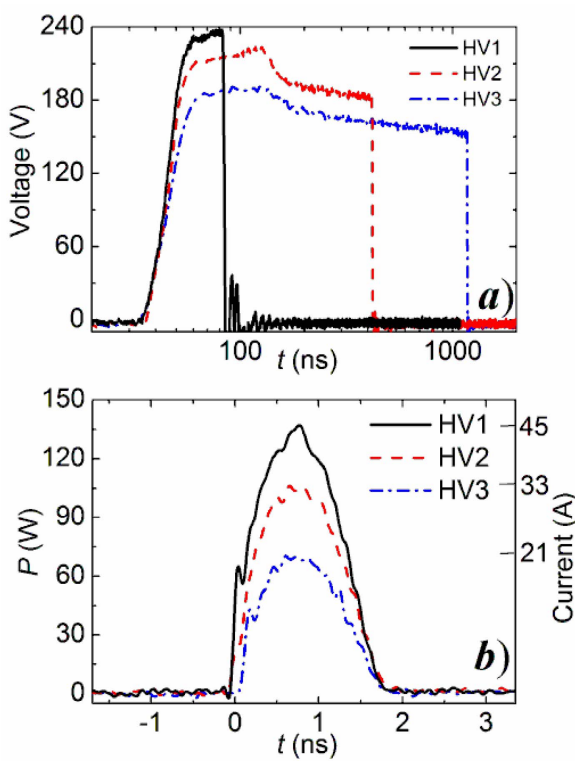

Fig. 3. (a) Experimental voltage across the S-diode and (b) optical power of LD for three different voltages of HV supply. The 0 ns on the time scale in Fig. 3(b) corresponds to 82, 415, and 1171 ns for curves HV1, HV2, and HV3 in Fig. 3(a), respectively.
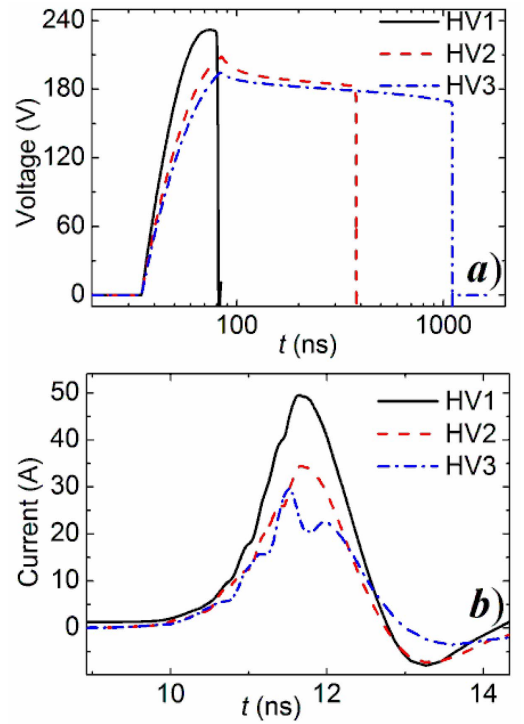

Fig. 4. (a) Experimental voltage across the S-diode and (b) current for three different voltages of HV supply. The $10 \mathrm{~ns}$ on the time scale in Fig. 4(b) corresponds to 80, 376, and 1093 ns for curves HV1, HV2, and HV3 in Fig. 4(a), respectively.

photoconductive semiconductor switch (PCSS) [19] (HG photoconductive switch). However, Vainshtein et al. [5], [6], [19] failed to address deep level recharging and its contribution to switching progress. In this article, TCAD-embedded deep level models were tested through a specially designed avalanche $S$ diode and follow-up calculations (details of the model will be presented in Section IV). The calculation results are presented in Fig. 4. The simulation circuit was changed since the Simulation Program With Integrated Circuit Emphasis (SPICE) parameters of diodes LD and VD were unknown [Fig. 2(a)]. Here, the laser diode was replaced by resistor $R 0=1 \Omega$.

Fig. 4 shows that TCAD-embedded models enable to correctly describe experimental dependences. Similar to the experiment, the calculations show an increased switching 
voltage and a reduced switching delay under a voltage ramp rise in the same orders of magnitude. Moreover, there is a twofold increase in the current pulse amplitude (the shape of the current pulse differs from the optical pulse, which is due to the laser gain-switching effect in the experiment). Apparently, it is not sensible to perfectly fit experimental and theoretical dependencies. This is due to the fact that exact values of many parameters are not known (Sections IV-VI will demonstrate that deep level recharging and switching behavior are sensitive to the carrier cross sections of deep levels and local concentration of deep levels in the filament region; both parameters are quite difficult to measure and control technologically).

\section{Avalanche S-Diode Model}

\section{A. CFD Switching}

The $S$-diode model was developed based on the previously described mechanisms [5], [6]. According to these, a switching procedure occurs through the filament. The remaining area of the superfast switch plays a significant role to ensure switching, since it is a source of leakage current at the preswitching stage (thanks to the large area) and a source of charges to ensure fast feeding of the filament channel at the stage initiating superfast switching [6]. Thus, the model includes two structures with similar doping parameters but different areas $\left(1.5 \mathrm{~mm}^{2}\right.$ for the diode over the whole area (WD) and $300 \mu \mathrm{m}^{2}$ for the filament-channel diode (FD), Fig. 5, inset). The filament area chosen is the same as for the previously investigated GaAs ABJT [6], since the dimensions of the active region of devices are similar (the thickness of the active region is $30-40 \mu \mathrm{m}$, the area is about $1 \mathrm{~mm}^{2}$ ). This article explores deep level recharging rather than the dynamics of superfast switching. The former basically takes much longer. Hence, with a view to reducing the calculation time, the switching progress was simulated following a simplified procedure, namely, through a 1-D case with a grid size of 7-20 nm. Also, we did not use realistic dependence of electron mobility on the electric field for the hot carriers in the field $E>200 \mathrm{kV} / \mathrm{cm}$ [5], [6]. Embedded TCAD model for electron mobility was instead used for this purpose:

$$
\mu(E)=\frac{\mu_{n}+\left(v_{s} / E\right) \cdot\left(E / E_{0}\right)^{4}}{1+\left(E / E_{0}\right)^{4}}
$$

where $\mu_{n}$ is the low-field electron mobility, $v_{s}$ is the saturation velocity, $E_{0}=4 \mathrm{kV} / \mathrm{cm}$.

This circumstance led to a relatively slow switching of the voltage across the structure, which lasted more than $1 \mathrm{~ns}$. The width of CFDs was relatively large, and its amplitude was small (less than $350 \mathrm{kV} / \mathrm{cm}$, Fig. 5). This is consistent with previously presented results from [5], [19]. The model used (1) is a fairly reasonable approach for preswitching simulation, since during the deep level recharging, the maximum electric field remains sufficiently low $(E<200 \mathrm{kV} / \mathrm{cm})$.

\section{B. Role of Electron Traps in S-Diode Switching Process}

Further parameters of the model were specified by way of solving two problems that had not been reported in the literature before:

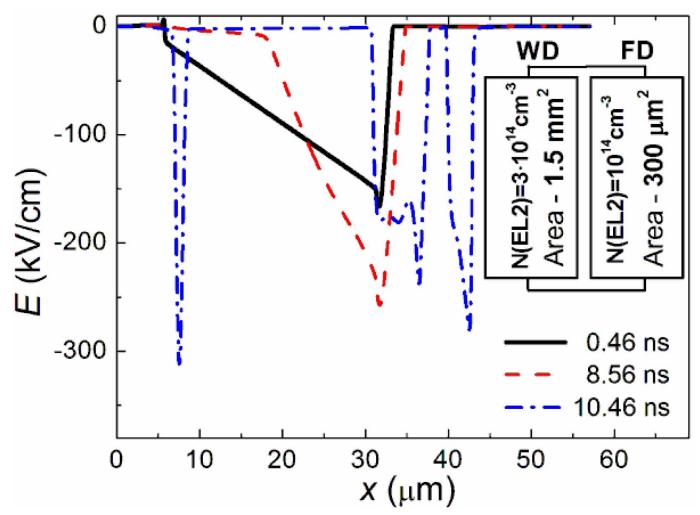

Fig. 5. Simulated electric field profiles for FD corresponding to transient presented in Fig. 4(b) (curve HV1). Inset shows sketch of simulated S-diode structure (WD and FD).

1) The elimination of huge leakage currents (several amperes) at the voltage ramping stage, which were observed during the simulation (Fig. 6, curve w/o EL2). The rationale for a lower level of these currents in the experiment (the leakage is generally less than $0.1-0.5 \mathrm{~A}$ in the experiment).

2) The selection of an inhomogeneity type in the S-diode structure, which leads to earlier fast switching of FD prior to slow switching of WD during the simulation.

The first problem results from the presence of two electron-hole junctions in the $S$-diode, which are connected in the opposite direction $\left(n^{+}-\pi\right.$ and $\left.\pi-n^{0}\right)$. When the $n^{+}$contact is under negative potential, the $n^{+}-\pi$-junction injects electrons into the $\pi$-layer, where these charge carriers drift to the reverse-biased $\pi-n^{0}$-junction. This causes a leakage current in the preswitching stage. During the simulation, this current can be reduced given the presence of effective electron traps in the $\pi$-layer. It is well known that the most efficient trapping centers of electrons in GaAs are the EL2-centers (a deep donor associated with the $\mathrm{As}_{\mathrm{Ga}}$ structural defect) [20]. The EL2-trap electron cross section can reach $3 \cdot 10^{-13} \mathrm{~cm}^{2}$ in the electric field of about $10 \mathrm{kV} / \mathrm{cm}$ [21], [22]. Based on the calculations, such a strong electric field exists in the $\pi$-layer at the preswitching stage due to the recharging inertia of deep Fe centers. The concentration of EL2-centers varies widely in different GaAs crystals (up to $2 \cdot 10^{16} \mathrm{~cm}^{-3}$ in the Czochralski-grown GaAs [23]). A relatively low concentration of EL2-centers, up to $3 \cdot 10^{14} \mathrm{~cm}^{-3}$, was found to be present in the HVPE-grown GaAs layers [24]. This value was used in the simulation for the WD.

The other problem is likely to be solved by assuming the presence of inhomogeneities that manifest themselves as a dislocation network in GaAs [25]. Large-scale fluctuations can be described as a set of dislocation cells sized $0.1-1 \mathrm{~mm}$. The concentration of EL2-centers varies by a factor of 2-4 from the center to the boundary of each cell [25]. In this case, the most natural solution was to assume that the concentration of EL2-centers in the region of the filament channel is lower than the one in the WD. A series of numerical experiments brought about a decision to use the concentration of EL2centers of $10^{14} \mathrm{~cm}^{-3}$ for the FD. This value provided good 


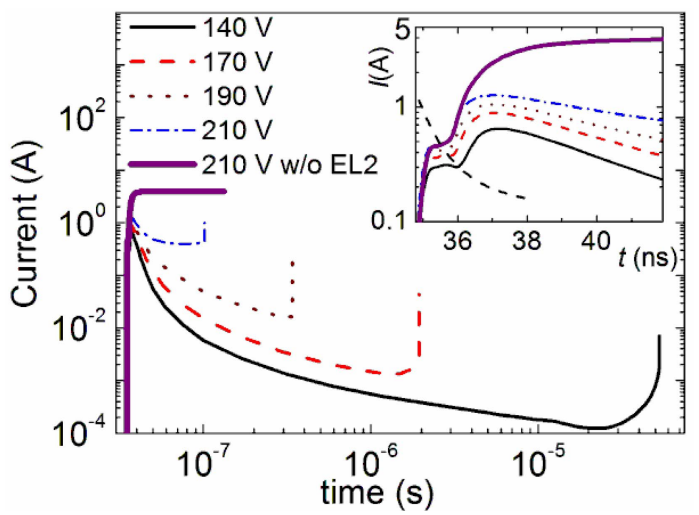

Fig. 6. Simulated current wave forms for different high-voltages of $\mathrm{HV}$ supply $\left(\sigma_{n}=3 \cdot 10^{-19} \mathrm{~cm}^{2}, \sigma_{p}=1.5 \cdot 10^{-16} \mathrm{~cm}^{2}\right)$.

agreement between the experimental and calculated results (Figs. 3 and 4).

\section{Deep Level Recharging at the Delay Stage}

At the switching delay stage, the Fe and EL2-centers are recharged in the $\pi$-layer. The dynamics of recharging determines electric field redistribution in the structure and, subsequently, determines the switching delay. The generation-recombination rate through both centers is defined by the following expression:

$$
R^{j}=\frac{N^{j} v_{n} v_{p} \sigma_{n}^{j} \sigma_{p}^{j}\left(n p-n_{i}^{2}\right)}{v_{n} \sigma_{n}^{j}\left(n+n^{j}\right)+v_{p} \sigma_{p}^{j}\left(p+p^{j}\right)}
$$

where $v_{n}$ and $v_{p}$ are the thermal velocities of electrons and holes, $n$ and $p$ are the electron and hole concentrations, $N$ is the trap concentration, $\sigma_{n}$ and $\sigma_{p}$ are the electron and hole capture cross sections, $n^{j}$ and $p^{j}$ are the electron and hole concentrations when the Fermi level $F$ is equal to trap energy level, $j$ denotes the trap level type (Fe or EL2).

In addition to thermal generation through deep centers, the model takes into account two reservoirs of nonequilibrium charge carriers, namely: 1-injection of electrons from a forward-biased $n^{+}-\pi$-junction; 2-avalanche generation and injection of holes from a reverse-biased $\pi-n^{0}$-junction. Field dependence of avalanche rate was calculated with the expression

$$
G_{A v a}^{h}=\alpha(E) v_{p} p, \alpha(E)=\alpha_{0} \cdot \exp \left(-E_{1} / E\right)
$$

where $\alpha_{0}=1.34 \cdot 10^{6} \mathrm{~cm}^{-1}, E_{1}=4 \cdot 10^{5} \mathrm{~V} / \mathrm{cm}$.

The electron population of $\mathrm{Fe}$ centers $f$ is a number between 0 and 1 , which changes, once caused by the capture and emission of electrons and holes

$$
\partial f / \partial t=(1-f) \cdot c_{C}-f \cdot e_{C}-f \cdot c_{V}+(1-f) \cdot e_{V}
$$

where $c_{i}$ denotes an electron capture rate for an empty trap, and $e_{i}$ denotes an electron emission rate for a full trap, $i$ denotes a process in the conduction band $(C)$ or valance band $(V)$

$$
\begin{aligned}
& c_{C}=\sigma_{n} v_{n} n \\
& c_{V}=\sigma_{p} v_{p} p \\
& e_{C}=\sigma_{n} v_{n} n^{j} \\
& e_{V}=\sigma_{p} v_{p} p^{j} .
\end{aligned}
$$

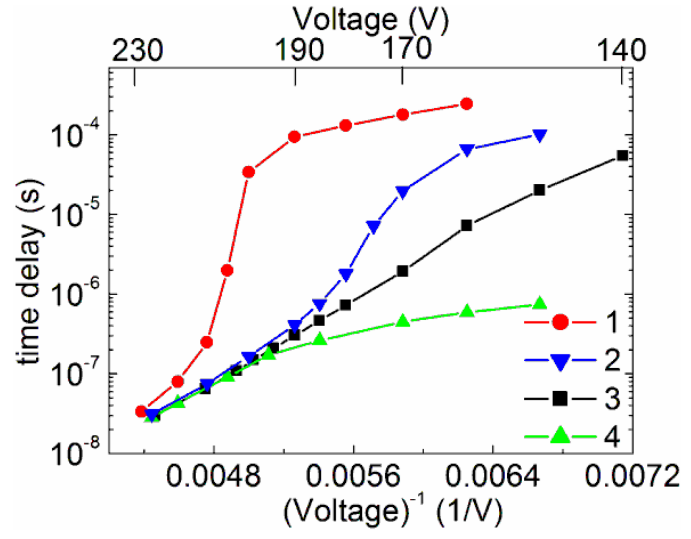

Fig. 7. Calculated dependences of time delay on voltage for different electron and hole capture cross sections $\left(\mathrm{cm}^{2}\right): 1-\sigma_{n}=3 \cdot 10^{-17}, \sigma_{p}=$ $1.5 \cdot 10^{-16} ; 2-\sigma_{n}=3 \cdot 10^{-19}, \sigma_{p}=1.5 \cdot 10^{-14} ; 3-\sigma_{n}=3 \cdot 10^{-19}, \sigma_{p}=$ $1.5 \cdot 10^{-16} ; 4-\sigma_{n}=3 \cdot 10^{-18}, \sigma_{p}=1.5 \cdot 10^{-16}$

Delay formation was studied based on the circuit shown in Fig. 2(b). Unlike the case described in Section III, in this case, the switch $S W$ was closed by a long control pulse that lasted $1 \mathrm{~s}$, which made it possible to form a stepwise voltage pulse across the capacitance $C 1$. This circuit is convenient for analyzing the processes to take place in the structure since the voltage remains time-constant until the $S$-diode switches [13] (however, in practice, this circuit is not applicable, since it requires a low pulse repetition rate).

\section{Simulation Results}

Fig. 6 shows $S$-diode current dependences for different stepwise voltage pulses $\left(V_{\mathrm{HV}}=V=140-225 \mathrm{~V}\right)$. In the voltage ramp region, a displacement current is accompanied by the injection of electrons from the $n^{+}$-layer into the $\pi$-region. For this reason, at the voltage ramping stage, the current increases to $0.1-1$ A (subject to $d V / d t$ ). The current subsequently drops at a constant bias voltage on the $S$-diode. Upon reaching certain conditions in the structure, a fast switching begins, characterized by a sharp current increase. The time interval between the moment the step voltage is supplied and the moment the $S$-diode switches on is referred to as the time delay $t_{d}$ at a given $\mathrm{HV}$ voltage $V_{\mathrm{HV}}$. The $t_{d}\left(1 / V_{\mathrm{HV}}\right)$ relationship calculated for various electron and hole capture cross sections are shown in Fig. 7. It can be seen that for small values of $1 / V_{\mathrm{HV}}$ (large applied voltages), all the curves calculated for different capture cross sections converge to the same exponential dependence $t_{d}=t_{0} \cdot \exp \left(V_{0} / V_{\mathrm{HV}}\right)$, where $t_{0}=7 \cdot 10^{-14} \mathrm{~s}$ and $V_{0}=2.9 \mathrm{kV} / \mathrm{cm}$. Once the voltage decreases, the curves diverge and can be described by a weaker exponential dependences with a value of $V_{0}=0.9 \mathrm{kV} / \mathrm{cm}$ at low voltages.

Some authors have already reported seeing dependences 1-3 in the experiments: either a single exponential dependence in a wide range of applied voltages (dependence 3, [13], [26]) or curves with a jump (dependences 1-2 [27]). Given that dependence 4 has not been found in the experiments, it will not be addressed in further analysis. 


\section{Discussion}

The simulation results in Section III show that the model developed describes the experimental data quite accurately (Fig. 3 and 4$)$. The functional $t_{d}\left(1 / V_{\mathrm{HV}}\right)$ relationship explicitly indicates that the $S$-diode model is accurate as well (Figs. 6 and 7). It is clear that Fe capture cross sections do not change in different $S$-diode samples described in the literature [13], [26], [27]. All changes to occur in the experimental $t_{d}\left(1 / V_{\mathrm{HV}}\right)$ relationship should be explained by the difference in $S$-diode configurations (difference in doping level and thickness of $\pi$-layer).

The $t_{d}\left(1 / V_{\mathrm{HV}}\right)$ relationships with a jump (Fig. 7, curves 1-2) have not been explained before. The exponential $t_{d}\left(1 / V_{\mathrm{HV}}\right)$ relationship (Fig. 7, curve 3) was viewed to be due to the fact that impact-ionization-induced holes were trapped by negatively charged $\mathrm{Fe}$ centers in the $n^{0}$-layer [13]. The rest of the article will show that holes are transported fast to the $\pi$-layer after avalanche generation, against the $n^{0}$-layer direction. Thus, the holes cannot be effectively trapped in the $n^{0}$-layer.

The electric field profiles show that irrespective of the conditions $\left(t_{d}, \sigma_{p}\right.$, and $\left.\sigma_{n}\right)$, the CFD switching mechanism is triggered as soon as a certain critical maximum field is reached $E_{c} \approx 180-240 \mathrm{kV} / \mathrm{cm}$ (the maximum electric field is localized at the $\pi-n^{0}$ junction). The said $E_{c}$ is possible only within a certain time delay $t_{d}$, since the field profile is reconstructed over time with inertia. Shortly after the voltage is biased, the electric field penetrates the entire $\pi$-layer depth as the uncompensated concentration of ionized $\mathrm{Fe}$ acceptors is low in this layer (in equilibrium $N_{\mathrm{Fe}}^{(-)}-N_{d}^{(+)} \approx 10^{11} \mathrm{~cm}^{-3}$ ). Then, several mechanisms are activated at once, thereby recharging the $\mathrm{Fe}$ centers in the $\pi$-layer and removing the field from the side of $n^{+}-\pi$ junction to the side of $\pi-n^{0}$ junction. Thus, the space charge region (SCR) is narrowed in the $\pi$-layer over the time interval before switching. In this case, it is obvious that with a constant voltage at the $S$-diode, the maximum electric field will increase at the $\pi-n^{0}$ junction up to the critical $E_{c}$. Then, sufficient avalanche generation will cause an intensive double injection regime (holes from the reverse biased $\pi-n^{0}$ junction and electrons from the forward biased $n^{+}-\pi$ junction) and initiate the CFD mechanism in a way similar to that for GaAs ABJT [5].

A series of numerical calculations performed in the work shows that the two exponential $t_{d}\left(1 / V_{\mathrm{HV}}\right)$ relationships can be conditioned by the two different SCR narrowing mechanisms. The jump of $t_{d}\left(1 / V_{\mathrm{HV}}\right)$ curve is due to the transition from one mechanism to another. With low time delays, the holes being trapped by negatively charged $\mathrm{Fe}$ ions near the $n^{+}-\pi$ junction are significant for SCR narrowing. With high time delays, the ionization of neutral $\mathrm{Fe}$ atoms in the depth of $\pi$-layer causes slower field reconstruction. Let us consider each mechanism in more detail.

\section{A. Short-Term Delay Mechanism (High Bias Voltage)}

Let us consider the case of a high bias voltage, e.g. $V_{\mathrm{HV}}=190 \mathrm{~V}$ (Figs. 6 and 7, curve 3). The biasing starts at 35 ns (Fig. 8, inset). The electric field profiles for different

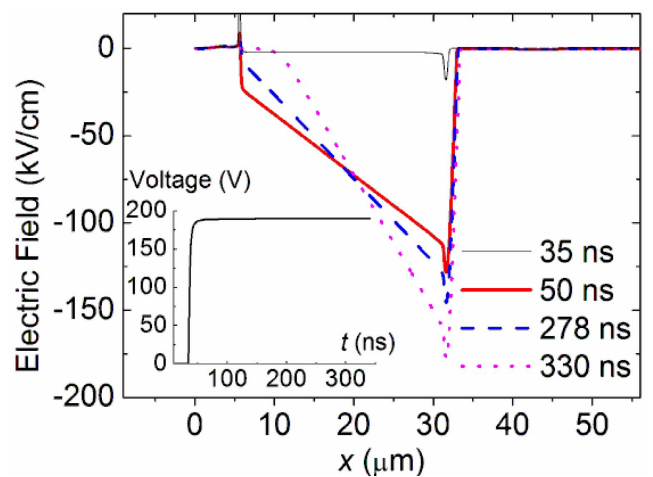

Fig. 8. Simulated electric field profiles for FD corresponding to transient presented in Fig. $6(190 \mathrm{~V})$. Inset shows voltage waveform across the S-diode.

time instants are presented in Fig. 8. The field is nonuniform just after the shift $(50 \mathrm{~ns})$ since electrons are injected into the $\pi$-layer from the $n^{+}$-layer (in contrast, in the $p^{+}-\pi-n^{0}-n$ structure, free from electron injection, the field is uniform in the $\pi$-layer).

A large bias voltage applied accelerates an electron injection current in the first stage of delay. Fig. 9(a) and (b) shows the concentration profiles for electrons and holes at different time instants. At the initial stage, the electron concentration sharply increases throughout the entire $\pi$-layer (from $10^{3}$ to $10^{14} \mathrm{~cm}^{-3}$ after $3 \mathrm{~ns}$ ) and the hole concentration reduces by two orders. In addition, electrons and holes intensely recombine near the $n^{+}-\pi$ junction over the entire time interval before switching, coupled with avalanche generation of holes that are "instantly" transferred to that recombination region. The fact that electrons and holes are supplied fast to the recombination region brings about an increased concentration of carriers near the $n^{+}-\pi$ junction (left) and overfilled negatively charged Fe deep centers. In this case, deeper EL2-centers are most crucial in the recombination process, while their overfilling (that, by all means, occurs) does not play a significant role in the formation of SCR, since EL2 concentrations are 500 times lower than those of Fe centers.

The rate at which the holes are trapped near the $n^{+}-\pi$ junction is determined by the capture cross sections (4). The hole cross sections for acceptor centers are generally much higher than those for electrons (the same is true for all curves in Fig. 7). Therefore, confronted with an excess of nonequilibrium charge carriers, the most important process is filling $\mathrm{Fe}$ atoms with holes. They grow in number near the $n^{+}-\pi$ junction by four orders relative to the equilibrium value (for equilibrium holes $p_{0} \approx 10^{11} \mathrm{~cm}^{-3}$ ). Over time, the concentration of neutral $\mathrm{Fe}$ centers will increase near the $n^{+}-\pi$ junction [Fig. 9(c)], which is likely to enhance the uncompensated positive charge of the shallow donors. This positive charge is neutralized by the electrons injected from the $n^{+}-\pi$ junction within a short-term diffusion-drift equilibrium (Maxwell relaxation time). This is the pattern to form a dynamic quasi-neutral region of $n$-type conductivity near the $n^{+}-\pi$ junction. Once formed, the $n$-type quasi-neutral region with electron concentrations of about $10^{14}-10^{15} \mathrm{~cm}^{-3}$ makes the field to be removed from the region near the $n^{+}-\pi$ junction 

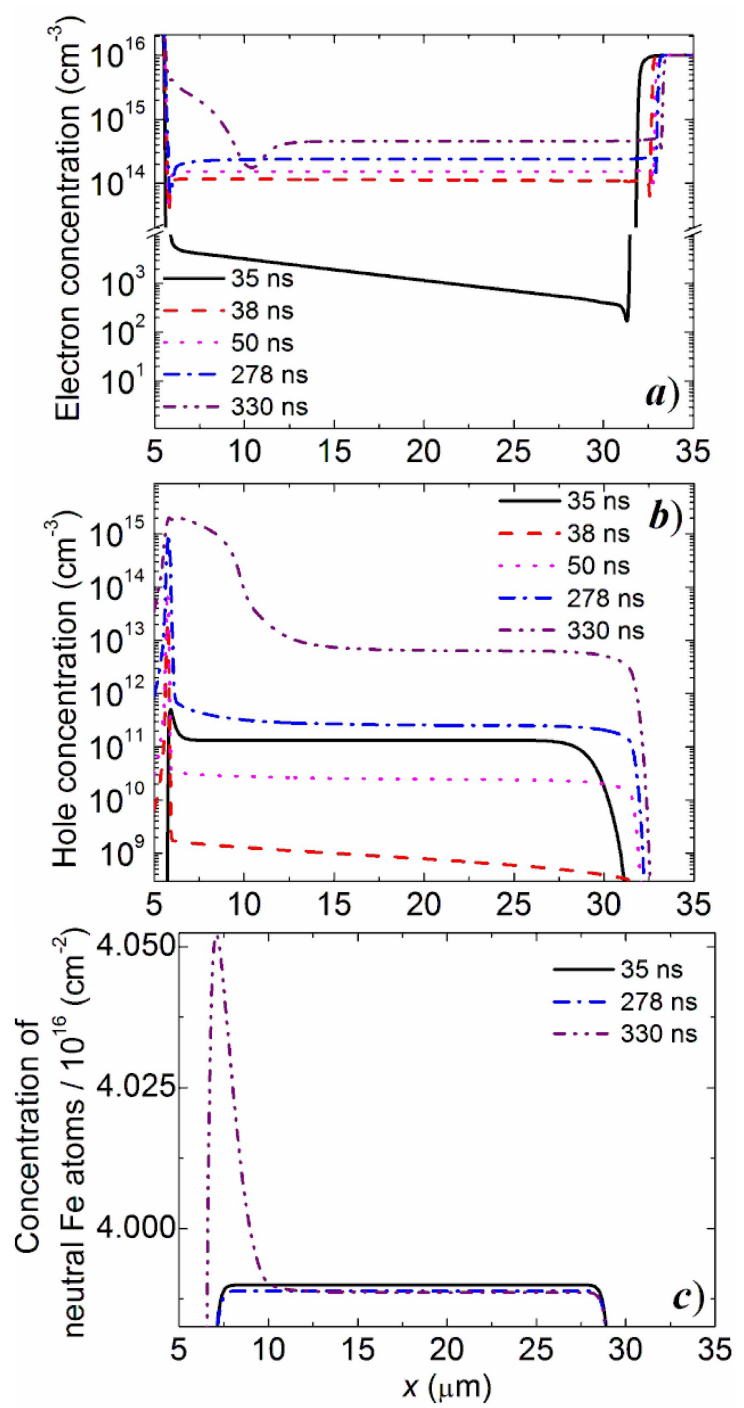

Fig. 9. Simulated profiles of electron concentration (a) hole concentration, (b) neutral Fe atoms concentration, and (c) for FD corresponding to transient presented in Fig. 6 (190 V).

and the SCR to be narrowed until the structure is switched on. The concentration of ionized (as well as neutral) $\mathrm{Fe}$ atoms in the $\pi$-layer depth remains unchanged throughout the entire time interval before switching [Fig. 9(c)]. Thus, there are no other significant capture (4.1) or emission (4.3), (4.4) processes in the said time delay mechanism.

Given the above conditions, the boost voltage increases the injection level. As a consequence, the hole concentration in the recombination region will rise and the hole capture time will go down $\left(\tau_{\mathrm{cp}}=1 / c_{\mathrm{V}}, 4.2\right)$. Thus, the delay time decreases with increasing voltage (Fig. 7, curve 3, $\left.V_{\mathrm{HV}}=190-225 \mathrm{~V}\right)$.

\section{B. Long-Term Delay Mechanism (Low Bias Voltage)}

Let us consider the case of a low bias voltage, e.g. $V_{\mathrm{HV}}=$ $140 \mathrm{~V}$ (Figs. 6 and 7, curve 3). The calculation shows, like the previous case, the SCR narrows with time. The field distribution before the switching does not qualitatively differ from the previous case. Following this switching delay mode, the double injection level is insufficient for a significant
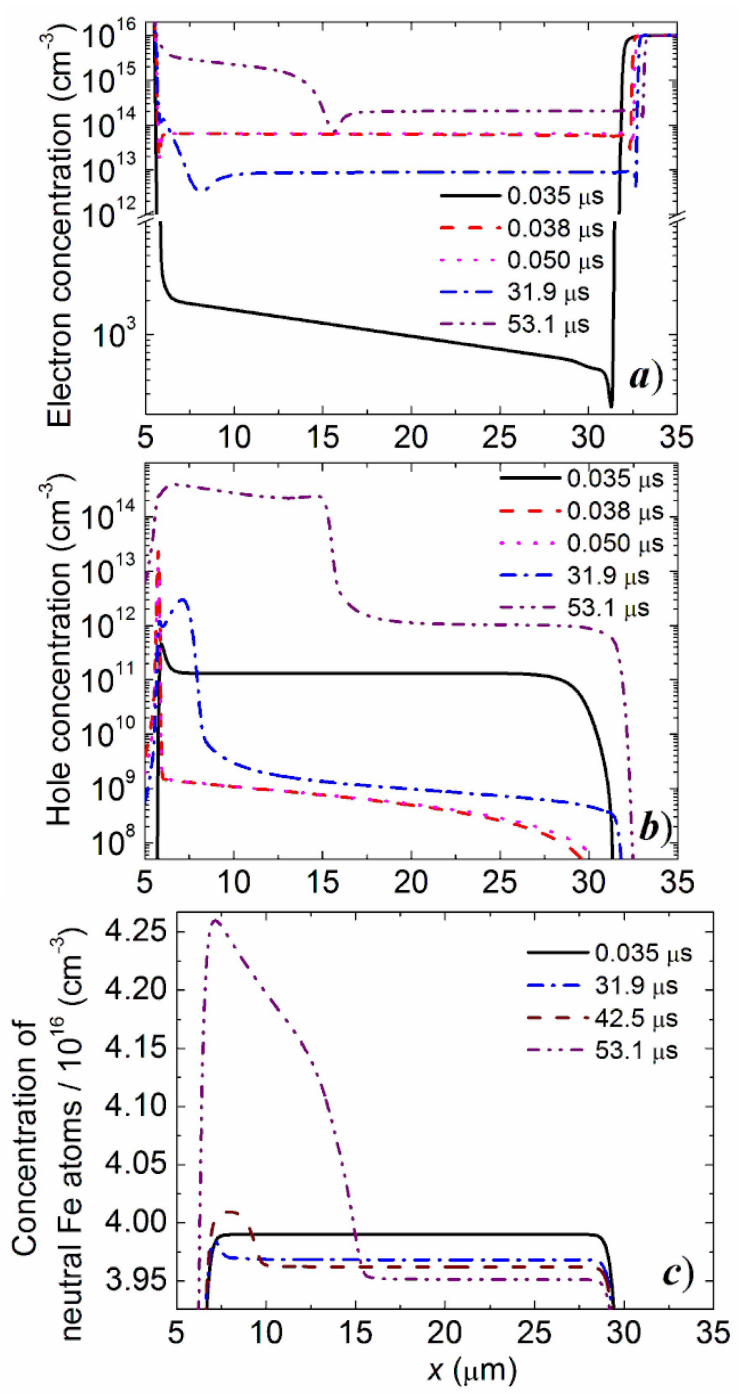

Fig. 10. Simulated profiles of electron concentration (a) hole concentration, (b) neutral Fe atoms concentration, and (c) for FD corresponding to transient presented in Fig. 6 (140 V).

concentration of electrons and holes near the $n^{+}-\pi$ junction [Fig. 10(a) and (b)]. Based on the calculations, the hole concentration $(p)$ in this region increases by no more than two orders and then decreases (holes are accumulated just at the end of the delay, before switching, due to the process described in Section IV. A, Fig. 10(b), $t=53.1 \mu \mathrm{s}$ ). A pure analytical estimate shows that the hole capture at the Fe centers takes $100-1000$ times longer than the previous case $\left(\tau_{\mathrm{cp}}=1 / c_{\mathrm{V}}=\right.$ $\left.\left(\sigma_{p} v_{p} p\right)^{-1}\right)$. However, over such a long time, other processes have time to occur, thus leading to SCR narrowing.

As long as the delay lasts, a negative charge of the Fe centers accumulates in the $\pi$-layer depth (the concentration of ionized $\mathrm{Fe}$ centers increases from $10^{11}$ up to $4 \cdot 10^{14} \mathrm{~cm}^{-3}$, the concentration of neutral $\mathrm{Fe}$ atoms decreases by $4 \cdot 10^{14} \mathrm{~cm}^{-3}$, Fig. 10(c). In this case, accumulation can be due to the capture of electrons from the conduction band (4.1) and the thermal emission of holes into the valence band (4.4). According to the expressions (4.1) and (4.4) for the electron concentration of $10^{13}-10^{14} \mathrm{~cm}^{-3}$, the $c_{\mathrm{C}}=30-300 \mathrm{~s}^{-1}$, and the $e_{\mathrm{V}}=30 \mathrm{~s}^{-1}$. Obviously, both mechanisms can contribute 
to the ionization of $\mathrm{Fe}$ atoms. Thus, as soon as the charge accumulates in traps, the SCR narrows until the injection level is sufficient enough to initiate the intense capture of holes near the $n^{+}-\pi$ junction. This, in turn, leads to the growth of neutral Fe atoms in this region [Fig. 10(c)], this faster mechanism is described in Section VI-A).

Additional calculations under similar conditions for the $p^{+}$ $\pi-n^{0}-n$ structure (in the absence of injected electrons) show that the time required for SCR narrowing increased by two orders, as compared to the $n^{+}-\pi-n^{0}-n$ structure. This indicates a stronger electron capture effect (4.1), as compared to hole emission (4.4).

Finally, if the voltage increases, the injection level increases sequentially. Accordingly, the effective electron capture time will decrease $\left(\tau_{\mathrm{cn}}=1 / c_{\mathrm{C}}, 4.1\right)$. Thus, the delay takes less time, provided that the voltage increases (Fig. 7 , curve $3, V_{\mathrm{HV}}=$ 140-160 V).

\section{CONCLUSION}

This article describes the effect of switching delay and the dynamic control over the switching voltage of superfast GaAs switches doped with a deep level impurity (avalanche Fe-doped $S$-diodes). Based on the example of a nanosecond LiDAR transmitter circuit for ADAS, the authors demonstrate the possibility of adjusting current pulse amplitude in the range from 20 to $45 \mathrm{~A}$. The amplitude is adjusted by changing the HV constant voltage.

Simulating the physical processes to exist in the avalanche $S$-diode prior to switching enables to show that the switching delay time is caused by the three deep level recharging processes, namely: hole capture and emission, as well as electron capture. Besides, there are two reservoirs of nonequilibrium carriers in the $S$-diode structure to be concerned with, namely: avalanche generation of holes and electron injection. Thus, deep levels are commonly recharged in the double injection mode.

The simulation results were analyzed to outline two mechanisms for the formation of the switching delay:

1) the capture of impact-ionization induced holes at negatively ionized Fe centers and the formation of the n-type quasi-neutral region near the electron injector $\left(n^{+}-\pi-\right.$ junction);

2) the accumulation of electrons at neutral Fe centers in the entire $\pi$-region and the formation of SCR with an increased concentration of negative charge.

Both mechanisms lead to the narrowing of the SCR at the delay stage (preswitching) until the critical field is reached in the avalanche region. Once available, the field facilitates the superfast switching following the mechanism of CFDs.

The multiplicity of processes determining the evolution of delay time makes it difficult to describe the delay mechanism and predict the value of dynamic switching voltage analytically. Nevertheless, qualitative reasoning suggests that, for fixed delays, an increase in dynamic switching voltage is possible due to an increase in the $\pi$-region thickness, since this reduces the injection level. A more detailed analysis requires further experiments and modeling to study the impact of $S$-diode parameters (layer thickness and doping level) on the dynamic switching voltage.

\section{REFERENCES}

[1] Operating the Pulsed Laser Diode SPL LL90_3. Osram Opto Semiconductrors. Accessed: Nov. 15, 2018. [Online]. Available: https:// dammedia.osram.info/media/resource/hires/osram-dam-2496583/ Operating\%20the\%20pulsed\%20laser\%20diode\%20SPL\%20LL90_ 3.pdf

[2] S. O. Slipchenko et al., "High peak optical power of 1 ns pulse duration from laser diodes-low voltage thyristor vertical stack," Opt. Express, vol. 27, no. 22, pp. 31446-31455, 2019, doi: 10.1364/OE.27.031446.

[3] J. Glaser, "How GaN power transistors drive high-performance lidar: Generating ultrafast pulsed power with GaN FETs," IEEE Power Electron. Mag., vol. 4, no. 1, pp. 25-35, Mar. 2017, doi: 10.1109/MPEL. 2016.2643099.

[4] A. Liero, A. Klehr, T. Hoffmann, T. Prziwarka, and W. Heinrich, "GaN laser driver switching 30 a in the sub-nanosecond range," in Proc. 46th Eur. Microw. Conf. (EuMC), London, U.K., Oct. 2016, pp. 1389-1392, doi: 10.1109/EuMC.2016.7824612.

[5] S. N. Vainshtein, V. S. Yuferev, and J. T. Kostamovaara, "Analyses of the picosecond range transient in a high-power switch based on a bipolar GaAs transistor structure," IEEE Trans. Electron Devices, vol. 52, no. 12, pp. 2760-2768, Dec. 2005, doi: 10.1109/TED.2005.859660.

[6] S. N. Vainshtein, V. S. Yuferev, J. T. Kostamovaara, M. M. Kulagina, and H. T. Moilanen, "Significant effect of emitter area on the efficiency, stability and reliability of picosecond switching in a GaAs bipolar transistor structure," IEEE Trans. Electron Devices, vol. 57, no. 4, pp. 733-741, Apr. 2010, doi: 10.1109/TED.2010.2041281.

[7] V. Palankovski, S. Vainshtein, V. Yuferev, J. Kostamovaara, and V. Egorkin, "Effect of hot-carrier energy relaxation on main properties of collapsing field domains in avalanching GaAs," Appl. Phys. Lett., vol. 106, no. 18, May 2015, Art. no. 183505, doi: 10.1063/ 1.4921006 .

[8] I. A. Prudaev, M. G. Verkholetov, A. D. Koroleva, and O. P. Tolbanov, "Charge carrier transport and deep levels recharge in avalanche S-Diodes based on GaAs," Tech. Phys. Lett., vol. 44, no. 6, pp. 465-468, Jun. 2018, doi: 10.1134/s106378501806007x.

[9] I. A. Prudaev et al., "The mechanism of superfast switching of avalanche $S$-diodes based on GaAs doped with $\mathrm{Cr}$ and $\mathrm{Fe}$," IEEE Trans. Electron Devices, vol. 65, no. 8, pp. 3339-3344, Aug. 2018, doi: 10.1109/TED.2018.2845543.

[10] I. A. Prudaev, S. S. Khludkov, M. S. Skakunov, and O. P. Tolbanov, "Switching avalanche S-diodes based on GaAs multilayer structures," Instrum. Exp. Techn., vol. 53, no. 4, pp. 530-535, Jul. 2010, doi: $10.1134 / \mathrm{S} 002044121004010 X$.

[11] S. S. Khludkov, "Gallium arsenide avalanche s-diodes," Sov. Phys. J., vol. 26, no. 10, pp. 928-937, Oct. 1983, doi: 10.1007/BF00896647.

[12] J. B. Rice and L. E. Nickels, "Commutation dv/dt effects in thyristor three-phase bridge converters," IEEE Trans. Ind. Gen. Appl., vol. IGA-4, no. 6, pp. 665-672, Nov. 1968, doi: 10.1109/TIGA.1968.4180963.

[13] P. Y. Beloborodov, O. P. Tolbanov, and S. S. Khludkov, "The influence of deep level recharging processes on avalanche breakdown delay of GaAs $\pi-v$-n structures compensated with iron," Sov. Phys. Semicond., vol. 22, no. 4, pp. 755-757, 1988.

[14] S. S. Khludkov and O. P. Tolbanov, "Semiconducting structures and devices based on gallium arsenide with deep centers," Russian Phys. J., vol. 35, no. 9, pp. 806-814, Sep. 1992, doi: 10.1007/BF00560054.

[15] S. M. Sze and K. K. Ng, Physics of Semiconductor Devices, 3rd ed. Hoboken, NJ, USA: Wiley, 2007.

[16] SPL DS90A_3 Chip Nanostack Pulsed Laser Diode. Osram Opto Semiconductrors. Accessed: Dec. 17, 2019. [Online]. Available: https:// dammedia.osram.info/media/resource/hires/osram-dam-7209242/SPL\% 20DS90A_3_EN.pdf

[17] I. V. Grekhov and A. F. Kardo-Sysoev, "Formation of subnanosecond current drops due to the delayed avalanche breakdown of $\mathrm{Si}$ p-n junctions," Sov. Tech. Phys. Lett., vol. 5, no. 15, pp. 950-953, Aug. 1979.

[18] P. Rodin, U. Ebert, A. Minarsky, and I. Grekhov, "Theory of superfast fronts of impact ionization in semiconductor structures," J. Appl. Phys., vol. 102, no. 3, Aug. 2007, Art. no. 034508, doi: 10.1063/1.2767378.

[19] L. Hu, J. Su, Z. Ding, Q. Hao, and X. Yuan, "Investigation on properties of ultrafast switching in a bulk gallium arsenide avalanche semiconductor switch," J. Appl. Phys., vol. 115, no. 9, Mar. 2014, Art. no. 094503, doi: 10.1063/1.4866715. 
[20] E. F. Schubert, Doping in III-V Semiconductors. New Providence, NJ, USA: AT\&T Bell Laboratories, 1993, doi: 10.1017/ CBO9780511599828.

[21] L. L. Bonilla, P. J. Hernando, M. Kindelan, and F. Piazza, "Determination of EL2 capture and emission coefficients in semi-insulating n-GaAs," Appl. Phys. Lett., vol. 74, no. 7, pp. 988-990, Feb. 1999, doi: $10.1063 / 1.123432$.

[22] M. Rogalla and K. Runge, "Formation of a quasi-neutral region in Schottky diodes based on semi-insulating GaAs and the influence of the compensation mechanism on the particle detector performance," Nucl. Instrum. Methods Phys. Res. A, Accel., Spectrometers, Detectors Associated Equip., vol. 434, no. 1, pp. 44-56, Sep. 1999, doi: 10.1016/S01689002(99)00431-3.

[23] D. E. Holmes, R. T. Chen, and J. Yang, "EL2 distributions in doped and undoped liquid encapsulated czochralski GaAs," Appl. Phys. Lett. vol. 42, no. 5, pp. 419-421, Mar. 1983, doi: 10.1063/1.93949.
[24] K. L. Schulte and T. F. Kuech, "A model for arsenic anti-site incorporation in GaAs grown by hydride vapor phase epitaxy," J. Appl. Phys., vol. 116, no. 24, Dec. 2014, Art. no. 243504, doi: 10.1063/ 1.4904745 .

[25] M. Müllenborn, H. Ch Alt, and A. Heberle, "Annealing behavior of deep-level defects in semi-insulating gallium arsenide studied by photoluminescence, infrared absorption, and resistivity mapping," J. Appl. Phys., vol. 69, no. 8, pp. 4310-4317, Apr. 1991, doi: 10.1063/1.348405.

[26] V. V. Kopyev, T. E. Smirnova, V. L. Oleynik, and I. A. Prudaev, "The influence of the deep level type on a switching time delay of GaAs avalanche S-diodes," in Proc. J. Phys., Conf., vol. 1124, no. 7, 2018 Art. no. 071013, doi: 10.1088/1742-6596/1124/7/071013.

[27] E. V. Bozhkova, D. D. Karimbayev, G. L. Prihod'ko, L. K. Tarasova, and S. S. Khludkov, "The electrical charachteristics of the S-diodes based on GaAs doped with iron impurity," Electron. Eng. Semicond. Devices, vol. 143, no. 8, pp. 59-65, 1980. 